\title{
Energy-Efficient Algorithms for $k$-Barrier Coverage In Mobile Sensor Networks
}

\author{
D. Ban, W. Yang, J. Jiang, J. Wen, W. Dou
}

\author{
Dongsong Ban, Wei Yang, Jie Jiang, Jun Wen, Wenhua Dou \\ National University of Defense Technology \\ School of Computer \\ Changsha, Hunan, P.R.China \\ E-mail: \{dsban,weiyang,jiejiang,junwen,whdou\}@nudt.edu.cn
}

\begin{abstract}
Barrier coverage is an appropriate coverage model for intrusion detection by constructing sensor barriers in wireless sensor networks. In this paper, we focus on the problem how to relocate mobile sensors to construct $\mathrm{k}$ sensor barriers with minimum energy consumption. We first analyze this problem, give its Integer Linear Programming(ILP) model and prove it to be NP-hard. Then we devise an approximation algorithm AHGB to construct one sensor barrier energy-efficiently, simulations show that the solution of AHGB is close to the optimal solution. Based on AHGB, a Divide-and-Conquer algorithm is proposed to achieve k-barrier coverage for large sensor networks. Simulations demonstrate the effectiveness of the Divide-and-Conquer algorithm.

Keywords: k-barrier coverage, energy-efficient relocation, mobile sensor network
\end{abstract}

\section{Introduction}

Monitoring and surveillance are very important applications of wireless sensor networks, such as detecting the intruders when they cross international borders, or detecting the spread of pollutant when sensors are deployed around critical regions(chemical plants,etc.) [1]. Barrier coverage which is achieved by barriers of sensors, is known to be an appropriate model of coverage for these applications. Different from the full coverage [2] where the goal is to achieve coverage for all points in the surveillance field, barrier coverage is unnecessary to cover every point in the field, thus barrier coverage requires much fewer sensors than full coverage.

Every sensor node is assumed to be stationary in most of barrier coverage literatures[1-5]. However, there are two problems for constructing sensor barriers in a randomly deployed stationary sensor network:1) The stationary network may contain gaps, as a result, constructing sensor barriers becomes impossible. 2) To avoid gaps, it will waste many sensor nodes by increasing the deployment density. To tackle these problems, we can utilize mobile sensors. After initial random deployment, a mobile sensor communicates with others to obtain the information of networks, and compute its new position for relocation, then relocate itself to new position. Therefore, it will avoid gaps in the sensor network with mobile sensors and guarantee to construct sensor barriers with much fewer sensors than stationary networks.

For mobile sensors, it is a prominent problem that how to construct $k$ sensor barriers with the minimum energy consumption. In barrier coverage model, any individual sensor cannot locally determine whether the given network provides k-barrier coverage or not [2]. However, if using global information, much communication overhead and computation cost will be brought, and it is unrealistic for large scale sensor networks. Thus, it is challenging to devise the efficient algorithm for constructing k-barriers in large scale networks with low communication and computation cost.

This paper is focused on how to achieve k-barrier coverage by the relocation of mobile sensors with the minimum energy consumption. We present an energy-efficient algorithm to construct 
1-barrier coverage, and a Divide-and-Conquer algorithm to construct k-barrier coverage in large scale sensor networks.

\section{Related Work}

Kumar et al. [2] first introduce the notion of weak barrier coverage and strong barrier coverage, and in [2] [3] the critical conditions of weak and strong barrier coverage in a randomly deployed stationary sensor network are derived. Chen et al. [1] introduce L-local barrier coverage, and devise a local algorithm for providing barrier coverage. For line-based deployed sensor network, Saipulla et al. [4] establish a tight lower-bound for the existence of barrier coverage. By exploiting collaborations and information fusion between neighboring sensors, Yang et.al [5] propose a centralized algorithm to find the smallest sensor set which can information-cover the barrier. All of the above works are focused on how to achieve barrier coverage in stationary network.

Recently, some research works are investigated for barrier coverage in mobile sensor network. Bhattacharya et al. [6] consider the problem how to optimally move sensors from the interior to the perimeter of a simple polygon region for detecting intruders. Yang [7] employs a game theoretic approach to study the sensor movement strategy to defend against barrier intrusions. Shen et al. [8] propose a centralized algorithm CBarrier and a distributed algorithm DBarrier to achieve 1-barrier coverage with mobile sensors. However neither of the proposed algorithms in [8] is energy-efficient because all sensors in the network are required to move. All aforementioned works only consider 1-barrier coverage problem with mobile sensors, and do not provide the effective solution for k-barrier coverage, which is important to enhance the successful probability of movement detection. Our work can achieve k-barrier coverage energy-efficiently with mobile sensors.

\section{Problem Statement}

$M$ sensors are randomly deployed in a two-dimensional rectangular strip $A$ with the width $w$ and the length $l . S=\left\{s_{1}, s_{2} \ldots, s_{M}\right\}$ denotes the set of all sensors. The initial position of $s_{i}$ is $\left(x_{i}, y_{i}\right)$ and its relocated position is $\left(x_{i}^{\prime}, y_{i}^{\prime}\right)$. The sensing model is disk, each sensor has the identical sensing radius $R_{s}$ and can get its own geographic location information.

The movement of mobile sensors consumes much energy, thus how to relocate them with as less energy as possible becomes very important.And after relocating, the mobile sensors should achieve barrier coverage. We first formulate the problem how to achieve 1-barrier coverage with the minimum energy consumption as 1-BCMS (1-Barrier Coverage of Min-Sum of moving distance ) problem, which is referred as follows.

Definition 1 (1-BCMS). Given a surveillance field $A$ and a set of mobile sensors $\mathrm{S}$, find a subset $S_{c}$ in $S$ and the destination position $\left(x_{i}^{\prime}, y_{i}^{\prime}\right)$ of each sensor $s_{i}$ in $S_{c}$, such that the sum of moving distance of all relocating sensors is minimized, meanwhile, the relocating sensors construct one sensor barrier in $A$.

\section{Problem Analysis}

For simplicity, $A$ is divided into $N$ equal-sized grids, where $N=n_{l} \times n_{w}, n_{l}=\left\lceil l / 2 R_{s}\right\rceil$, $n_{w}=\left\lceil w / 2 R_{s}\right\rceil$. The set of central points of grids is $G=\left\{g_{1}, g_{2}, \ldots, g_{N}\right\}$, where $g_{i}$ denotes the central point of the $i^{\text {th }}$ grid. It is obvious that a grid can obtain 1-barrier coverage when just only one sensor is located at its central point. Thus, this paper assumes that the destination 
position of relocation of each sensor is selected from $G$. Under the above grid model, we call a sensor barrier as a grid barrier, which is shown in Figure 1. In a grid barrier, every sensor is located at the central point of a grid, and the distance between neighboring sensors is no more than $2 R_{s}$.

As shown in Figure 1, the surveillance field is 1-barrier covered after constructing a grid barrier with mobile sensors. The problem how to construct a grid barrier with the minimum moving distance could be formulated as 1-GBMS (1-Grid Barrier Min-Sum of moving distance) problem. Obviously, based on grid model, 1-GBMS problem is equivalent to 1-BCMS problem. Therefore, we study the 1-GBMS problem in the following.

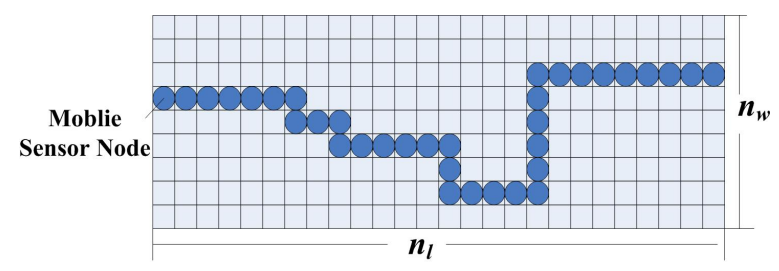

Figure 1: A grid barrier

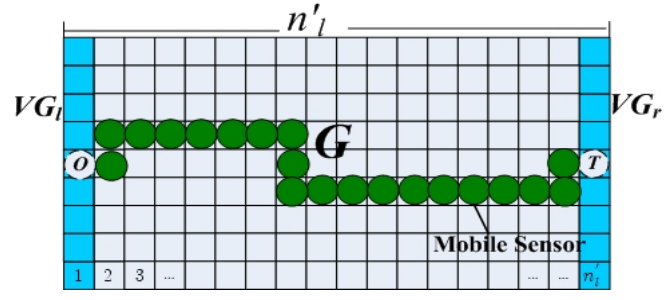

Figure 2: Region A after adding virtual vertices and virtual columns

Definition $2(1-G B M S)$. Given a set of mobile sensors $\mathrm{S}$ and the set $\mathrm{G}$ of central points of grids in a surveillance field $A$, find a subset $S_{g}$ in $S$ and the destination position $p_{i}$ from $G$ for each sensor $s_{i}$ in $S_{g}$, such that the sum of moving distance is minimized, meanwhile, the relocating sensors construct a grid barrier in $A$.

In this section, the Integer Linear Programming Model of 1-GBMS is derived. As shown in Figure 2, We first add two virtual columns of grids beside the leftmost and rightmost column, and two distinguished vertices $\mathrm{O}$ and $\mathrm{T}$ that represent the virtual origin and the virtual destination of any barrier, respectively. The set of grids in the left virtual column is denoted by $\mathrm{VG}_{l}$, and the set of grids in the right one is denoted by $V G_{r}$. $A G=G \cup V G_{r} \cup V G_{l}$ denotes the set of all grids, $A S=S \bigcup\{O, T\}$ denotes the set including all sensor nodes and two virtual vertices. We give sequence number to vertices in AS and AG, respectively. Specifically, The sequence number of $O$ is 1 , and that of $T$ is $M^{\prime}$, all sequence numbers of the actual sensors are given from 2 to $M^{\prime}-1$. All grids in $A G$ are given from 1 to $N^{\prime}$. $n_{l}^{\prime}$ denotes the number of grids in one row. Let $x_{i j}=1$, when the sensor $s_{i}$ moves to the grid $g_{j}$, otherwise $x_{i j}=0$. The distance between $s_{i}$ and $g_{j}$ is $d_{i j}$. The ILP model is described in Figure 3.

Constraint (2) and (3) enforce that a sensor is only allowed to move into at most one grid, and a grid is only allowed to be covered by at most one sensor. A barrier could be considered as a flow from $\mathrm{O}$ to $\mathrm{T}$. Constraint (4) constrains that the flow is 1, which entering a grid in leftmost column of $\mathrm{G}$ from virtual vertex $\mathrm{O}$, and constraint(5) guarantees that the flow leaving a grid in rightmost column of $G$ to $T$ is 1 . By flow conservation law, if one sensor moves into a grid $g_{j}$, there must exist two sensors in the four adjacent grids(left, right, top, bottom) of $g_{j}$ to make sure that the flow of entering $g_{j}$ and leaving $g_{j}$ are 1 , respectively. This constraint is enforced by constraint (6).

We show by Theorem 1 that the 1-GBMS problem is NP-hard by restrictions to the famous Knapsack Problem.

Theorem 3. 1-GBMS Problem is NP-hard.

Proof: Firstly, we make some restrictions of 1-GBMS problem as follows. 


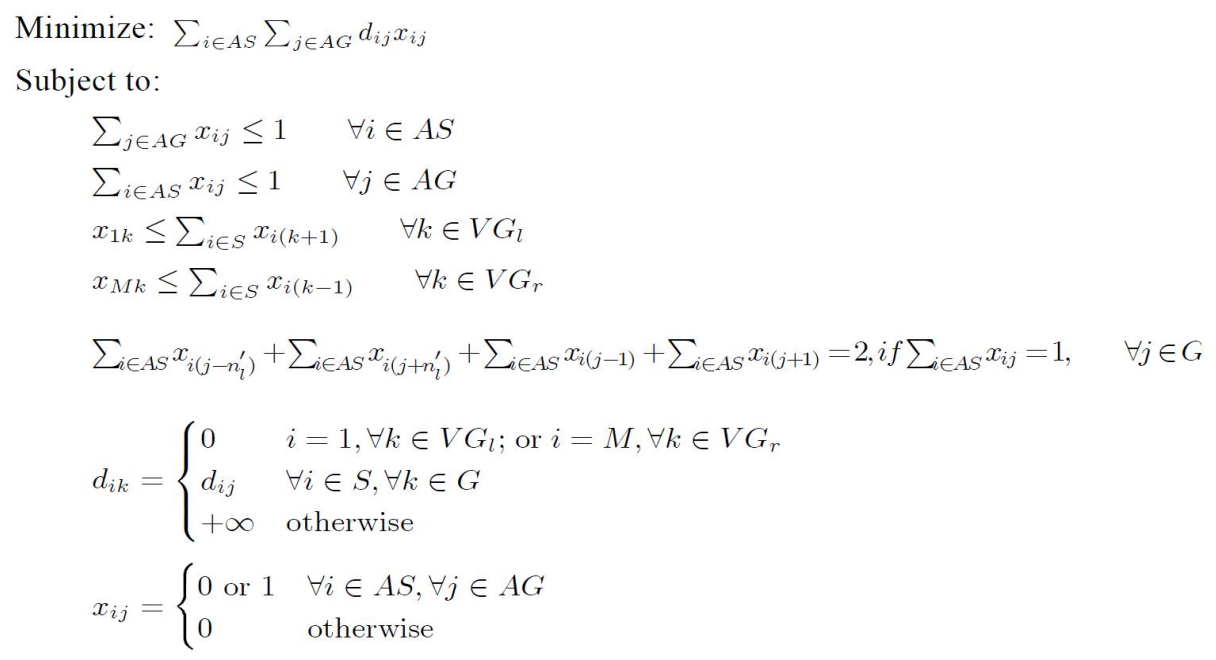

Figure 3: ILP Model

Restriction1: $\forall j, k \in G, \forall i \in S$, let $d_{i j}=d_{i k}$, i.e., for any sensor $s_{i}$, the distance between $s_{i}$ and any grid is equal.

Restriction2: The number of sensors that construct a grid barrier is no more than $K$. In original 1-GBMS problem, there is no restriction to this number.

According to Restriction1, the moving distance of a sensor is just related to its own position, i.e., if we select one sensor, the moving distance of this sensor is fixed. Thus, the sum of moving distance is just related to the set of selected sensors. We assume $c_{i}$ is the cost when $s_{i}$ moves to $g_{j}$, let $c_{i}=1 / d_{i}$, as a result, $c_{i}$ increases as $d_{i}$ decreases. Then 1 -GBMS problem is reduced as a new problem how to select no more than $K$ sensors from $S$, such that the sum of cost is maximized. The new problem formulation is shown as follows.

Maximize: $\sum_{i=1}^{M} c_{i} x_{i}$

Subject to:

$\sum_{i=1}^{M} 1 \times x_{i} \leq K$

$x_{i}=\{0,1\}, i=1,2,3, \ldots . . M$

(11) shows that the number of selected sensors is no more than $K$. This formulation is the same as Linear Programming Model of Knapsack Problem [9]. Since Knapsack Problem is NPhard,the original problem 1-GBMS is NP-hard.

The solution of 1-GBMS problem by ILP model is optimal, but it is polynomial unsolvable as the network size increases since it is NP-hard. Thus a polynomial approximation algorithm is required.

\section{AHGB Algorithm}

Under grid model, there are $\mathrm{n}_{w}$ rows of grids in $A$. If we deploy one sensor in each grid in a selected row, it is said to construct a horizontal grid barrier in $A$. As a result, $A$ is 1-barrier covered. Kumar [2] proved that by constructing horizontal grid barrier the fewest sensors are required to achieve 1-barrier coverage. Thus the mobile sensors needed to relocate for 1-barrier coverage by constructing horizontal grid barrier is fewest, and the sum of moving distance in this relocation method could be less than other method with great probability. Based on above observations, we propose an approximation algorithm AHGB(Approximate to Horizontal Grid 
Barrier), which mainly has two steps :1) Horizontal Grid Barrier Selection, which finds a row from $A$ as a horizontal grid barrier. 2) Optimal Movement, which finds the optimal movement strategy for relocating mobile sensors to the selected horizontal grid barrier, subject to minimize the sum of moving distance.

\subsection{Horizontal Grid Barrier Selection}

If each of the mobile sensors which construct the barrier moves to its nearest grid, the sum of moving distance is minimized. Based on the above idea, we propose HGBS approach. For any grid $g_{k}$, we first assign a weight to it. The weight is the distance between $g_{k}$ and the nearest sensor $s_{i}$ to it. Then we compute the sum of weight of each row, and find the row which has the smallest weight as barrier_position. The HGBS approach is described as shown in Figure.4.

\subsection{Optimal Movement}

If we select the $i^{\text {th }}$ row as the position of horizontal grid barrier by HGBS, the destination positions of mobile sensors needed to move are known. The destination positions are the $\boldsymbol{n}_{l}$ grids in $i^{\text {th }}$ row. Then we select $n_{l}$ nodes from $M$ sensors, and find the optimal movement strategy to relocate the selected sensors to the $\boldsymbol{n}_{l}$ grids in horizontal grid barrier. We call this problem as Optimal Movement Problem.

When we find the optimal movement strategy subject to minimize the sum of moving distance, we get a one-to-one matching between the selected sensors and grid positions with the minimum sum of weight. Thus Optimal Movement Problem is equivalent to Bipartite Weighted Matching Problem [9]. The Hungarian Method is known as an optimal solution to Bipartite Weighted Matching Problem, which can be used as the solution method for Optimal Movement Problem. The computation complexity of Hungarian is $\mathrm{O}\left(\mathrm{m}^{2} n\right)$ [9], $\mathrm{m}$ is the size of the set which has fewer nodes, and the size of the other set is $n$.

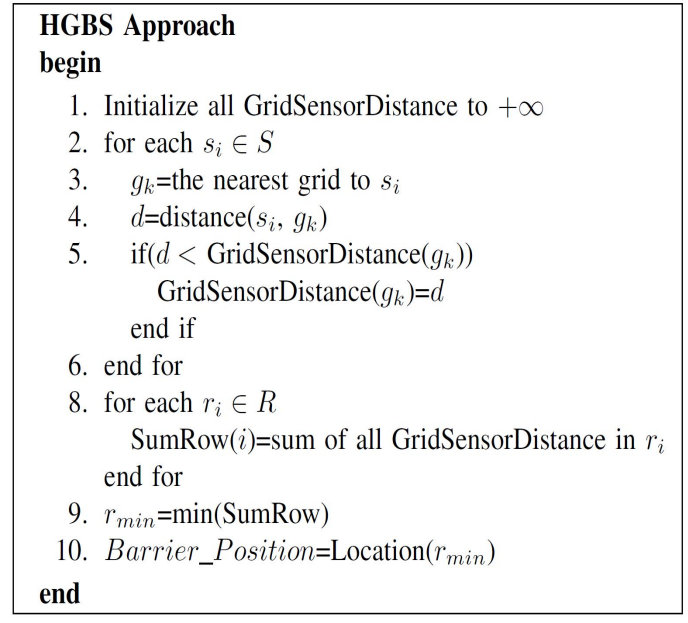

Figure 4: HGBS Approach

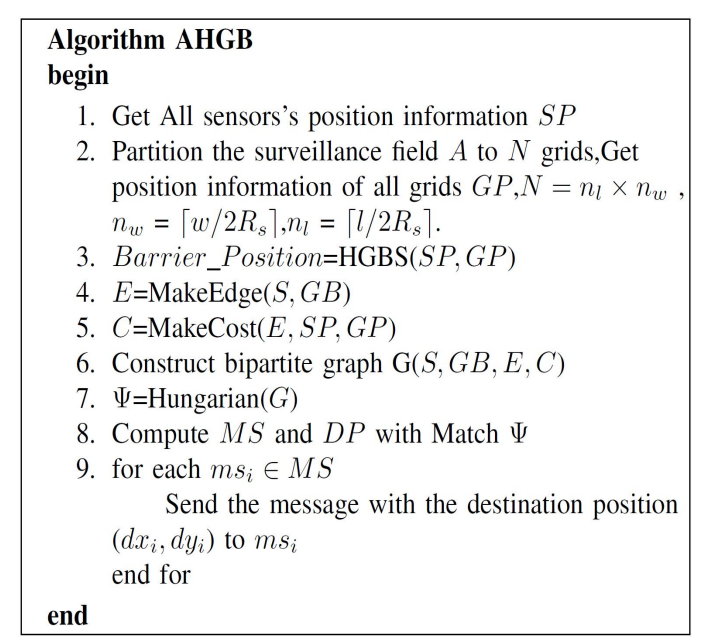

Figure 5: Algorithm AHGB

\subsection{AHGB Algorithm}

The AHGB Algorithm is summarized briefly as follows. First, we get the position information of all sensors(SP) and the position information of $\mathrm{N}$ grids (GP), and find the horizontal grid barrier by HGBS approach. Then we construct a weighted bipartite graph with SPand GB 
which denotes the set of grids in the selected horizontal grid barrier, and find the optimal matching by Hungarian Method to identify the sensors required to move(MS) and their destination positions(TP). At last,we send destination positions to identified sensors and move them to grids in horizontal grid barrier. The pseudocode of algorithm AHGB is shown in Figure 5.

The computation complexity of the step3 and step7 is $O(N)$ and $O\left(n_{l}^{2} M\right)$ [9], respectively. Since $n_{l}>n_{w}$, we can obtain $n_{l}^{2}>N$. Therefore, the computation complexity of AHGB is $\mathrm{O}\left(\mathrm{n}_{l}^{2} M\right)$. The communication overhead of AHGB is $\mathrm{O}(M)$, because we need to get the positions of all $M$ sensors at the initial phase as shown in step1. In section 7 , we show that the performance of AHGB is close to the optimal solution.

Algorithm AHGB can only construct one sensor barrier energy-efficiently, and it is not applicable for large scale sensor networks because it is centralized. In the next section, we present an efficient algorithm to achieve k-barrier coverage for large scale sensor networks.

\section{Divide-and-Conquer Algorithm}

$k$-barrier coverage is often required to guarantee to detect the moving target. According to [2], one sensor cannot locally determines whether the surveillance field is k-barrier covered or not. It is difficult to devise a distributed and energy-efficient algorithm for k-barrier coverage in large scale sensor networks. [8] presented a distributed algorithm DBarrier to construct 1-barrier coverage, which cannot provide the solution for k-barrier coverage. Furthermore, DBarrier is not energy-efficient since all sensors in the network are required to move iteratively. In this section, we propose a Divide-and-Conquer algorithm to achieve k-barrier coverage energy-efficiently for large scale sensor networks. The main idea of Divide-and-Conquer algorithm is described as follows:

1) $A$ is divided into $k \times v$ equal-sized subregions, the length of each subregion is $l_{s}=l / v$, the width is $w_{s}=w / k$.

2) In each subregion, algorithm AHVGB(Approximate to Horizontal and Vertical Grid Barrier) independently constructs a horizontal grid barrier and a vertical grid barrier.

3) We obtain k-barrier coverage in A when all AHVGB are finished. Figure. 6 illustrates that $A$ is 3 -barrier covered when the Divide-and-Conquer algorithm is finished.
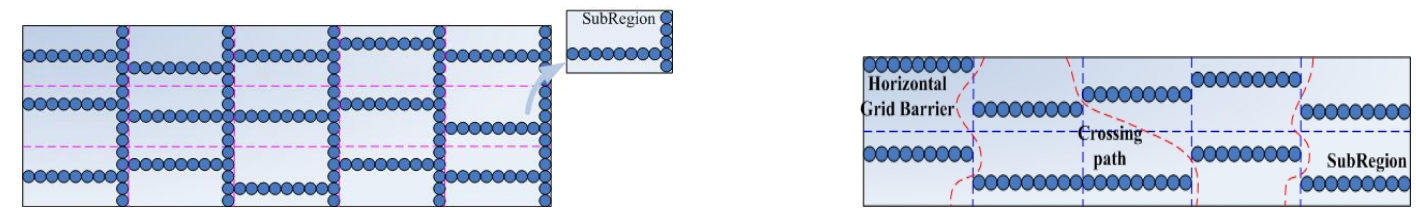

Figure 6: 3-barrier coverage when the Divide- Figure 7: Gaps may exist in sensor networks and-Conquer algorithm is finished

\subsection{AHVGB Algorithm}

As shown in Figure 7, there maybe exist a gap between two horizontal neighboring subregions. Thus, additional sensors are required to connect the two adjacent horizontal grid barriers to eliminate gaps. This section proposes a method which adds a vertical grid barrier between two adjacent subregions. Since the width of subregion is small, the sensors required to move for constructing vertical grid barrier is few.

We use AHVGB algorithm to construct a horizontal grid barrier and a vertical grid barrier in each subregion. In AHVGB algorithm, every subregion can independently construct barriers by 
local information in its own area without communication with other subregions. The AHVGB is improved from AHGB algorithm. Specifically, when horizontal grid barrier has been selected, AHVGB constructs the weighted bipartite graph with NewGB $=\mathrm{GB} \cup \mathrm{G}_{\mathrm{r}}$, but AHGB uses $\mathrm{GB}$, as shown in step6 in Figure 5. GB is the set of grids in the selected horizontal grid barrier, $\mathrm{G}_{\mathrm{r}}$ denotes the set of grids in the rightmost vertical column of $A$.

\subsection{Low Communication Overhead and Computation Cost}

In our Divide-and-Conquer algorithm, each subregion is only $\frac{1}{k \times v}$ of $A$. By dividing the large surveillance strip into small subregions, the message delay, communication overhead, and computation cost can be significantly reduced. The position information of a sensor node only need to be transmitted within the small subregion where the node is located, resulting in smaller delay and communication overhead compared with the whole network. The computation cost is also much lower since the number of nodes in each subregion is much less than that in the whole area.

\section{Performance Evaluation}

We conduct simulations by MATLAB7.0 to evaluate the performance of our algorithms. The sensing radius $R_{s}=2.5 \mathrm{~m}$. The mobile sensors are randomly deployed according to a Poisson point process.

In this section, we evaluate the performance of AHGB compared with the optimal solution computed by ILP model, which is polynomial unsolvable as the network size increases. In Figure 8 , sensors are initially deployed in a $15 \mathrm{~m} \times 500 \mathrm{~m}$ strip, it shows the comparison of the sum of the moving distance between AHGB and the optimal solution, as the number of sensors increases from 100 to 800 . The performance of AHGB is close to the optimal solution. In particular, the difference is only $4 \%$ when the sensor number is 300 .

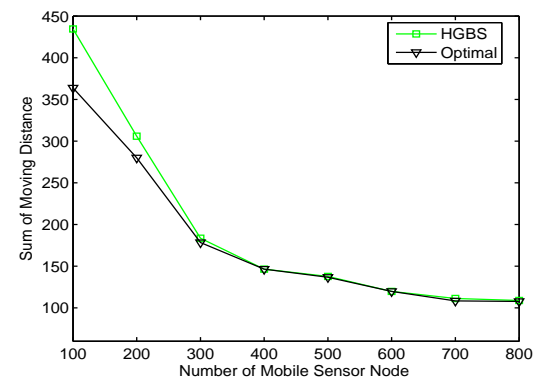

Figure 8: Sum of Moving Distance vs. Node number

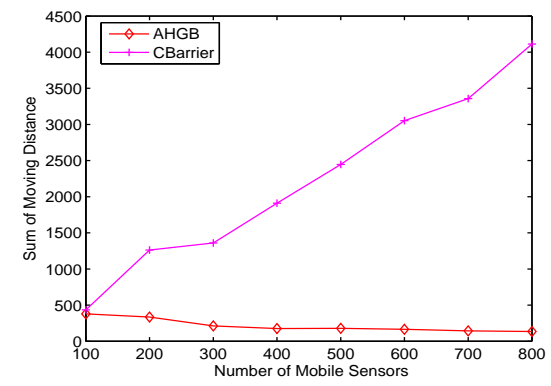

Figure 9: AHGB vs. CBarrier

We compare AHGB with CBarrier [8]which also achieves 1-barrier coverage. In Figure 9, the sum of moving distance by CBarrier increases fast as the number of sensors increases, but that of AHGB does not change much. The reason is that all sensors in the network are required to move in CBarrier, and only part of sensors is selected for relocation in AHGB .The experiment parameters of Figure 9 are the same as Figure 8.

Figure 10 shows the effectiveness of Divide-and-Conquer algorithm. There are many gaps in the network after initial deployment, and 3-barrier coverage is achieved, when the algorithm is finished. Sensors are initially deployed in a $60 \mathrm{~m} \times 160 \mathrm{~m}$ strip according to Poisson point process with the density 0.02 . 


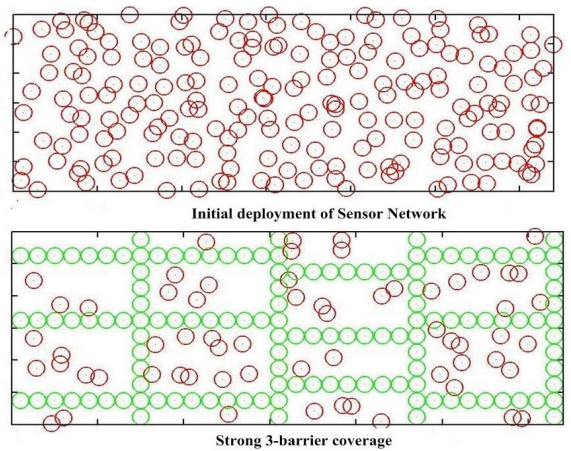

Figure 10: Effectiveness of Divide-and-Conquer algorithm

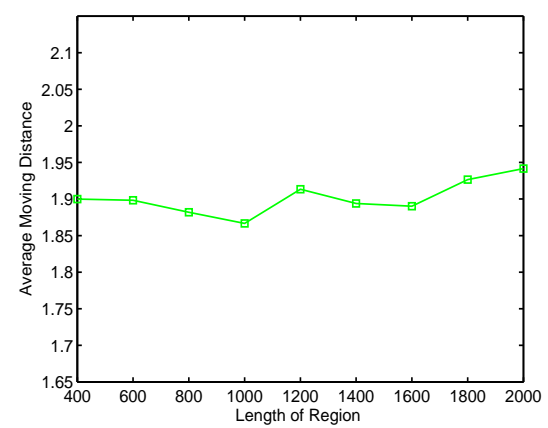

Figure 11: Average Moving Distance vs. Length of Region

As shown in Figure 11, the average moving distance of sensors does not change much, as we increase the length of strip with the constant deployment density 0.02. It implies that our Divideand-Conquer algorithm can be applicable to large scale sensor networks. The initial experiment parameters of Figure 11 are the same as Figure 10.

\section{Conclusions}

In this paper, we first formulate the problem how to construct 1-barrier coverage with the minimum energy consumption in mobile sensor network as 1-BCMS problem, and analyze it in detail. Then an energy-efficient algorithm for 1-barrier coverage and an energy-efficient Divideand-Conquer algorithm for k-barrier coverage are presented. At last, simulations demonstrate the effectiveness and energy-efficiency of the proposed algorithms. In the future, we will study how to achieve k-barrier coverage in hybrid sensor networks.

\section{Acknowledgments}

This work was supported in part by the National Natural Science Foundation of China under Grant No. 60603061, No.60903223

\section{Bibliography}

[1] Chen, S. Kumar and T. H. Lai. Designing Localized Algorithms for Barrier Coverage. In Proc. of Mobicom07, ACM, pp:63-74, 2007.

[2] S. Kumar, T. H. Lai and A. Arora. Barrier Coverage With Wireless Sensors. In Proc. of Mobicom05, ACM, pp:284-298, 2005.

[3] Benyuan Liu, Olivier Dousse and Jie Wang. Strong Barrier Coverage of Wireless Sensor Networks. In Proc. of Mobihoc08, ACM, pp:411-420, 2008.

[4] Anwar Saipulla, Benyuan Liu and Jie Wang. Barrier Coverage of Line-Based Deployed Wireless Sensor Networks In Proc. of INFOCOM09, IEEE, pp:127-135, 2009.

[5] Guanqun Yang and Daji Qiao. Barrier Information Coverage with Wireless Sensors.In Proc. of INFOCOM09, IEEE, pp:918-926, 2009 
[6] B. Bhattacharya, M. Burmester, Y. Hu, E. Kranakis and Q. Shi. Optimal Movement of Mobile Sensors for Barrier Coverage of a Planar Region. In Proc. of COCOA, pp:103-115, 2008 .

[7] Guanqun Yang, Wei Zhou and Daji Qiao. Defending Against Barrier Intrusions with Mobile Sensors. In Proc. of WASA0\%, pp: 113-120, 2007

[8] CX Shen, WF Cheng, XK Liao and SL Peng. Barrier coverage with mobile sensor. In Proc. of I-SPAN08, IEEE, pp.99-104, 2008.

[9] Eugene Lawler. Combinatorial Optimization:Networks and Matroids. Holt, Renehart and Winston Press, NewYork, 1976. 